\title{
Influência do calor armazenado no sistema solo-planta no balanço de energia em pomar de mangueiras ${ }^{1}$
}

\author{
Cícera J. R. Borges², Pedro V. de Azevedo², Vicente de P. R. da Silva², João H. B. da C. Campos², \\ Magna S. B. de Moura ${ }^{2}$, José M. Soares ${ }^{2} \&$ Bernardo B. da Silva ${ }^{2}$
}

\begin{abstract}
RESUMO
Neste estudo se objetivou a avaliação da contribuição do calor armazenado acima das placas de fluxo de calor no solo e no dossel vegetativo sobre os componentes do balanço de energia, no pomar de mangueiras. Os componentes do balanço de energia foram obtidos pelo método do balanço de energia com base na razão de Bowen. O teste t-Student foi usado para avaliar a existência de diferença significativa entre as médias dos componentes do balanço de energia, obtidas mediante as seguintes condições: (i) considerando-se o calor armazenado acima dos fluxímetros e (ii) desconsiderando-se o calor armazenado acima dos fluxímetros, cujos resultados indicaram que: 1) o efeito do calor armazenado no dossel vegetativo é irrelevante quando comparado com os outros componentes do balanço de energia em pomar de mangueiras; 2) o calor armazenado acima das placas de calor no solo não interfere significativamente no cálculo dos fluxos de calor sensível e latente nem na evapotranspiração da mangueira irrigada; 3) o fluxo de calor no solo, corrigido com o calor armazenado acima dos fluxímetros, difere estatisticamente a nível de $5 \%$ de probabilidade, daquele obtido sem a correção do fluxo.
\end{abstract}

Palavras-chave: evapotranspiração, razão de Bowen, fluxo de calor no solo

\section{Influence of the heat stored in the soil-plant system in the energy balance over a mango orchard}

\begin{abstract}
The objective of this study was to investigate the contribution of the heat storage above the soil heat flux plates and within the canopy upon the components of the energy balance in the mango orchard. The Bowen energy-ratio balance method was used for obtaining the energy balance components and evapotranspiration of the mango orchard. The tStudent test was applied to evaluate statistical significance of the difference between energy balance component averages under the following conditions: (i) considering and (ii) not considering the heat storage above the fluximeters. The results suggested that: 1) the canopy heat storage is irrelevant compared to the other energy balance components; 2) the above soil heat plates heat storage does not influence the sensible and latent heat fluxes nor the irrigated mango orchard evapotranspiration; 3) the soil heat flux, when corrected for the heat storage above the soil heat plates differs statistically, at the $5 \%$ probability level, from that obtained without correction.
\end{abstract}

Key word: evapotranspiration, Bowen ration, soil heat flux

1 Parte da Dissertação de mestrado da primeira autora apresentada ao Programa de Pós-Graduação em Meteorologia da UFCG.

2 UACA/UFCG. Av. Aprígio Veloso, 882, Bodocongó, CEP 58109-970, Campina Grande, PB, Fone: (83) 3310-1202. E-mail: cicerasc@yahoo.com.br; pvieira@dca.ufcg.edu.br; vicente@dca.ufcg.edu.br; jhbcampos@dca.ufcg.edu.br; magna@cpatsa.embrapa.br; monteiro@cpatsa.embrapa.br; bernardo@dca.ufcg.edu.br 


\section{INTRODUÇÃO}

O método do balanço de energia é utilizado, comumente, para se estimar a evapotranspiração de superfícies vegetadas e se baseia na análise do balanço energético dos ganhos e perdas de energia térmica radiativa, condutiva e convectiva por uma superfície evaporante. Próximo à superfície e de forma simplificada, o balanço de energia é representado pela partição do saldo de radiação $\left(R_{n}\right)$, nos seguintes fluxos: fluxo de calor no solo $(\mathrm{G})$, fluxo de calor sensível $(\mathrm{H})$ e em fluxo de calor latente (LE). Bowen (1926) desenvolveu o método do balanço de energia baseado no quociente entre os fluxos de calor sensível e latente, o qual combina as variáveis atmosféricas e a energia disponível na superfície evaporante. O conhecimento dos componentes do balanço de energia sobre superfícies cultivadas é de grande importância para a estimativa das perdas de água por evapotranspiração e caracterização do microclima local; além disso, ele pode contribuir para a utilização mais racional da água pelas culturas, cujas fases críticas de desenvolvimento são limitadas pelo fator hídrico.

O balanço de energia determinado através da razão de Bowen (BERB) é freqüentemente utilizado na quantificação dos fluxos de calor latente e sensível emitidos e/ou recebidos por superfícies vegetadas, em função dos gradientes verticais de pressão do vapor d'água e da temperatura do ar observados na camada de ar próxima à superfície do dossel vegetativo. O BERB tem sido muito empregado por vários pesquisadores na estimativa da evapotranspiração de culturas, devido à precisão e simplicidade operacional em relação aos demais métodos de estimativa da evapotranspiração, quando se dispõe da instrumentação necessária às medições locais e em alta freqüência do saldo de radiação e dos gradientes verticais de temperatura e pressão de vapor do ar (Trambouze et al., 1998; Azevedo et al., 2003; Silva et al., 2006); sua eficiência é maior em culturas de pequeno porte como, trigo e milho (Mokate et al., 1995; Cunha et al., 1996); mesmo assim, o BERB tem sido utilizado em culturas de grande porte porém com menor freqüência devido às seguintes restrições do método: i) a igualdade entre os coeficientes de transferência turbulenta de calor sensível $\left(K_{h}\right)$ e latente $\left(\mathrm{K}_{\mathrm{w}}\right)$ é válida apenas para condições de estabilidade atmosférica próximo à neutralidade, ii) as medidas de temperatura do ar e pressão de vapor d'água devem ser efetuadas dentro da camada limite superficial (vegetação-atmosfera), com ausência de gradientes horizontais, implicando na necessidade de um "fetch" (bordadura), adequado; este método funciona bem em condições úmidas, o que não ocorre em condições secas porque a razão de Bowen ( $\beta$ ) é, geralmente, alta e positiva; enquanto na presença de advecção de energia ela é negativa (Angus \& Watts, 1984). Apesar de representar não mais de $5 \%$ do saldo de radiação $\left(\mathrm{R}_{\mathrm{n}}\right)$, o fluxo de calor no solo $(G)$ deve ser bem representado, uma vez varia mais que $\mathrm{R}_{\mathrm{n}}$ à superfície; assim, para se obter boa representatividade do fluxo de calor no solo de uma área, faz-se necessária a instalação de vários fluxímetros para a medição do fluxo de calor no solo; em geral esses instrumentos são instalados em profundidades que variam de 2 a $10 \mathrm{~cm}$, dependendo dos objetivos do estudo. A partição do saldo de radiação em G está relacionada à cobertura vegetal, umidade do solo e a posição dos fluxímetros no solo, ou seja, o fluxo de calor no solo não pode ser medido diretamente na superfície do solo, pois o fluxímetro não pode ficar exposto diretamente à radiação solar (Silans et al., 1997); por este motivo, Stannard et al. (1994) sugeriram a utilização de pelo menos três sensores: um na área coberta pela vegetação, outro na área exposta e um terceiro na área intermediária, posicionado entre o solo exposto e a cobertura vegetal. Se o solo estiver totalmente coberto, a porcentagem de G gira em torno de 5 a 10\% do saldo de radiação $\left(\mathrm{R}_{\mathrm{n}}\right)$; caso o solo esteja coberto parcialmente, este valor aumenta bastante, podendo variar de 20 a $40 \%$ do saldo de radiação (Kustas et al., 2000).

A maioria dos trabalhos sobre balanço de energia, baseado na razão Bowen, não contabiliza o fluxo de calor armazenado no dossel da planta (Daamen et al., 1999; Azevedo et al., 2003); o argumento geralmente utilizado é que este componente do balanço de energia é muito pequeno em comparação com os demais fluxos do balanço de energia. Portanto, se espera que, se a planta tiver um dossel bastante desenvolvido, o calor armazenado por ela pode apresentar uma contribuição considerável no cálculo do balanço de energia; por outro lado, também em trabalhos com balanço de energia geralmente não se considera o calor armazenado acima dos fluxímetros; esses sensores devem ser instalados o mais próximo possível da superfície do solo, pois sua finalidade é medir o fluxo de calor que entra e sai do solo; entretanto, por falta de conhecimento os mesmos são comumente instalados a $2 \mathrm{~cm}$ de profundidade. Deste modo, pouco se sabe se este procedimento não conduz a erros apreciáveis no balanço de energia de culturas irrigadas; neste contexto, se objetivou, com o trabalho, investigar os efeitos do calor sensível armazenado no dossel das plantas e na camada de solo acima dos fluxímetros sobre o balanço de energia no sistema solo-vegetação.

\section{MATERIAL E MÉTODOS}

A pesquisa foi realizada em um pomar de mangueiras da variedade "Tommy Atkins", na fazenda Agropecuária Boa Esperança S.A. (9²0' S, 40²7' O e 375 m) localizada na BR 428, km 183, no município de Petrolina, PE, na região do Submédio rio São Francisco. De acordo com a classificação de Koppen, o clima da região do ensaio experimental é do tipo BSwh' (clima semi-árido), com período chuvoso de janeiro a abril. As variáveis meteorológicas da região apresentam os seguintes valores médios (Teixeira, 2001): temperatura do ar: $26,4{ }^{\circ} \mathrm{C}$; precipitação média anual: 567 mm; umidade relativa do ar: 63\%; média diária da evaporação do tanque Classe A: 7,4 $\mathrm{mm} \mathrm{d}^{-1}$; velocidade do vento a $2 \mathrm{~m}$ de altura: $193 \mathrm{~km} \mathrm{~d}^{-1}$ e insolação: 7,3 h.

O experimento foi instalado em um pomar de mangueiras (Mangifera indica L), com 12 anos de idade, plantado no espaçamento $5 \times 10$ m e irrigado por microaspersão. A parcela experimental foi irrigada com base no coeficiente de cultivo proposto por Azevedo et al. (2003). A coleta de 
dados ocorreu no período de março a agosto de 2005, envolvendo os diversos estágios fenológicos do ciclo produtivo da mangueira. Durante o período experimental se mantiveram todos os procedimentos de tratos culturais adotados pela fazenda, tais como: controle de pragas e doenças; capinas e adubação via água de irrigação.

\section{Instrumentação e coleta de dados}

No interior da parcela experimental se montou uma torre micrometeorológica para a instalação dos seguintes instrumentos: dois anemômetros de conchas (modelo 03001, marca Young), nos níveis de 0,5 e 1,9 m acima da copa das plantas, para medição da velocidade vento (V); dois radiômetros (modelo CM3, marca Kipp \& Zonen), a 1,95 m, para medições da radiação solar incidente (Ri) e refletida $(\mathrm{Rr})$ acima do dossel vegetativo; um saldo radiômetro (modelo NR lite, marca Kipp \& Zonen) posicionado sobre a copa das plantas para medição do saldo de radiação $\left(\mathrm{R}_{\mathrm{n}}\right)$; dois psicrômetros não-aspirados a base de cobre-constantan devidamente calibrados, para medição das temperaturas dos bulbos seco e molhado, situados a 0,5 e 1,9 m acima do dossel vegetativo e um sensor para medição da temperatura e umidade relativa do ar (modelo HMP45C, marca Campbell Scientific, Inc.).

Instalaram-se, no solo, dois fluxímetros (modelo HFT3, marca Campbell Scientific, Inc.) para medição do fluxo de calor no solo $(\mathrm{G})$, posicionados a $0,08 \mathrm{~m}$ de profundidade, distanciados 1,0 m do tronco da planta, no sentido latitudinal e longitudinal. Para cada fluxímetro, foram instalados dois termistores (modelo 107, marca Campbell Scientific, Inc.) para medição da temperatura do solo (Ts), localizados a 0,02 e 0,06 m de profundidade, logo acima dos fluxímetros. Além disso, dois tubos de acesso foram utilizados para possibilitar a medição do conteúdo de umidade no solo, instalados a 0,20 m de cada ponto de observação, nas profundidades de 10, 20, 30, 40, 60 e 100 cm, utilizando-se sonda FDR (Frequency Domain Reflectometry, modelo PR1/6, marca Delta T).

O perfil vertical da temperatura do ar foi medido no interior do dossel de uma planta selecionada, por meio de termopares de cobre-constantan instalados a 1,0; 2,0 e 3,0 m acima da superfície do solo; todos os sensores foram conectados a um sistema automático de aquisição e armazenamento de dados (Datalogger CR 23X, Campbell Scientific, Inc.), programado para efetuar leitura dos sensores a cada cinco segundos e médias a cada 20 min. Todos os dados foram utilizados para elaboração mais completa possível do balanço de energia com base no método da razão de Bowen.

\section{Balanço de energia}

Obtiveram-se os componentes do balanço de energia sobre a vegetação por meio da equação do balanço de energia com base na razão de Bowen, de acordo com a seguinte expressão (Rosenberg et al., 1983):

$$
\mathrm{R}_{\mathrm{n}}+\mathrm{LE}+\mathrm{H}+\mathrm{G}+\mathrm{S}_{\mathrm{d}}+\mathrm{F}=0
$$

em que $R_{n}$ é o saldo de radiação, LE e H são os fluxos verticais de calor latente e sensível, respectivamente; $\mathrm{G}$ o fluxo de calor no solo, $\mathrm{S}_{\mathrm{d}}$ a energia armazenada no sistema solo- dossel vegetativo e F é a energia utilizada no processo fotossintético, a qual não foi considerada por representar menos de $2 \%$ do saldo de radiação (Heilman et al., 1994). Todos os termos da Eq. 1 foram expressos em $\mathrm{W} \mathrm{m}^{-2}$. O balanço de energia foi efetuado sobre o dossel do pomar de mangueiras, para um volume de controle compreendido entre a profundidade de $8 \mathrm{~cm}$ do solo e a altura do psicrômetro mais elevado (1,95 m acima do dossel do cultivo); consideram-se positivos os fluxos verticais que chegam e negativos os que saem do volume de controle.

\section{Fluxo de calor latente}

O fluxo de calor latente (LE), considerando-se o fluxo de calor no solo com correção $\left(G_{c c}\right)$ e sem correção $\left(G_{s c}\right)$ do calor armazenado acima dos fluxímetros, foi obtido substituindo-se a razão de Bowen ( $\beta=\mathrm{H} / \mathrm{LE})$ na Eq. 1 , ou seja:

$$
\begin{aligned}
& \mathrm{LE}_{1}=\frac{\mathrm{R}_{\mathrm{n}}+\mathrm{G}_{\mathrm{cc}}+\mathrm{S}}{1+\beta} \\
& \mathrm{LE}_{2}=\frac{\mathrm{R}_{\mathrm{n}}+\mathrm{G}_{\mathrm{sc}}+\mathrm{S}}{1+\beta}
\end{aligned}
$$

em que os subscritos 1 e 2 se referem às estimativas do fluxo de calor latente com base em medições no fluxo de calor sensível para o solo com e sem correção, respectivamente.

O termo $\beta$ foi obtido como:

$$
\beta=\frac{\mathrm{H}}{\mathrm{LE}}=\frac{\overline{\mathrm{P}}_{\mathrm{Cp}_{\mathrm{p}}}}{\mathrm{L} \in}\left(\frac{\mathrm{K}_{\mathrm{h}}}{\mathrm{K}_{\mathrm{w}}}\right) \frac{\partial \mathrm{T} / \partial \mathrm{Z}}{\partial \mathrm{e} / \partial \mathrm{Z}}
$$

em que $\overline{\mathrm{P}}$ é a pressão atmosférica média $(\mathrm{kPa}) ; \mathrm{K}_{\mathrm{h}}$ e $\mathrm{K}_{\mathrm{w}}$ são, respectivamente, os coeficientes de transferência nos processos de difusão turbulenta de calor sensível e latente, expressos em $\mathrm{m}^{2} \mathrm{~s}^{-1}$, e $\gamma$ o coeficiente psicrométrico $\left(\mathrm{hPa}{ }^{\circ} \mathrm{C}^{-1}\right)$, obtido pela expressão:

$$
\gamma=\frac{\overline{\mathrm{P}} \mathrm{C}_{\mathrm{p}}}{0,662 \mathrm{~L}}
$$

em que $\mathrm{C}_{\mathrm{p}}=1,0032 \mathrm{~J} \mathrm{~g}^{-1}{ }^{\circ} \mathrm{C}^{-1}$, representa o calor específico do ar a pressão constante, $\overline{\mathrm{P}}$ pressão atmosférica média à superfície $(\mathrm{hPa})$ e L o calor latente de vaporização $\left(\mathrm{MJ} \mathrm{g}^{-1}\right)$ obtido por (Wright, 1982) como:

$$
\mathrm{L}=2,487-0,51 \mathrm{~T}_{\mathrm{a}}
$$

em que $\mathrm{T}_{\mathrm{a}}$ é a temperatura do ar $\left({ }^{\circ} \mathrm{C}\right)$. Considerando-se a igualdade entre $\mathrm{K}_{\mathrm{h}}$ e $\mathrm{K}_{\mathrm{w}}$ e $(\partial \mathrm{T} / \partial \mathrm{Z}) /\left(\partial \mathrm{e}_{\mathrm{a}} / \partial \mathrm{Z}\right) \approx \Delta \mathrm{T} / \Delta \mathrm{e}_{\mathrm{a}}$; sendo $\Delta \mathrm{T}=\mathrm{T}_{2}-\mathrm{T}_{1}$ e $\Delta \mathrm{e}_{\mathrm{a}}=\mathrm{e}_{2}-\mathrm{e}_{1}$ as diferenças de temperatura do ar e pressão de vapor d'água na camada de ar acima da copa da árvore, respectivamente, a Eq. 4 foi simplificada para:

$$
\beta \approx \gamma \frac{\Delta \mathrm{T}}{\Delta \mathrm{e}_{\mathrm{a}}}
$$

Calculou-se a pressão parcial de vapor d’água em dois níveis acima da copa das plantas pela equação de Ferrel, da seguinte forma: 


$$
\mathrm{e}\left(\mathrm{t}_{\mathrm{a}}\right)=\mathrm{e}_{\mathrm{s}}\left(\mathrm{t}_{\mathrm{u}}\right)-0,00066\left(1+0,00115 \mathrm{t}_{\mathrm{u}}\right)\left(\mathrm{t}_{\mathrm{a}}-\mathrm{t}_{\mathrm{u}}\right) \overline{\mathrm{P}}
$$

em que $t_{u}$ e $t_{a}$ são as temperaturas dos bulbos molhado e seco, respectivamente, expressas em ${ }^{\circ} \mathrm{C}, \overline{\mathrm{P}}$ a pressão atmosférica média $(\mathrm{hPa})$ e $\mathrm{e}_{\mathrm{s}}\left(\mathrm{t}_{\mathrm{u}}\right)$ é a pressão de saturação do vapor d’água (hPa), que foi calculada pela equação de Tetens, apresentada por Rosenberg et al. (1983), como:

$$
e_{s}\left(t_{u}\right)=6,1078 \exp \frac{17,269 \cdot t_{u}}{237,3+t_{u}}
$$

Os valores de $\beta$ passaram por uma análise de consistência de dados conforme estabelecido por Unland et al. (1996); tal procedimento ocorreu quando $\beta$ se aproximou de -1 , resultando em valores incorretos de LE.

\section{Fluxo de calor sensível}

O fluxo de calor sensível $(\mathrm{H})$ foi obtido como resíduo da Eq. 1, ou seja:

$$
\begin{aligned}
& \mathrm{H}_{1}=\left(\mathrm{R}_{\mathrm{n}}+\mathrm{LE}_{1}+\mathrm{G}_{\mathrm{cc}}+\mathrm{S}\right) \\
& \mathrm{H}_{2}=\left(\mathrm{R}_{\mathrm{n}}+\mathrm{LE}_{2}+\mathrm{G}_{\mathrm{sc}}+\mathrm{S}\right)
\end{aligned}
$$

\section{Fluxo de calor no solo}

Os fluxos de calor no solo (G), ao nível de 0,08 m de profundidade, e o calor armazenado na camada de solo acima deste nível foram obtidos conforme (Kustas et al., 2000):

$$
\mathrm{G}=\mathrm{G}_{\mathrm{p}}+\frac{\Delta \mathrm{T}_{\mathrm{s}} \mathrm{C}_{\mathrm{s}} \mathrm{D}}{\mathrm{t}}
$$

em que $G_{p}$ é a medição de fluxo de calor no solo pelo fluxímetro, $\Delta \mathrm{T}_{\mathrm{s}}$ é a diferença média da temperatura do solo $\left({ }^{\circ} \mathrm{C}\right)$ durante o período de medição, $\mathrm{D}$ é a profundidade do fluxímetro, "t" é o intervalo da medição em minutos e $\mathrm{C}_{\mathrm{s}}$ é a capacidade calorífica volumétrica do solo, obtida pela expressão:

$$
\mathrm{C}_{\mathrm{s}}=\rho_{\mathrm{s}}\left(\mathrm{C}_{\mathrm{sd}}+\mathrm{C}_{\mathrm{sw}} \theta_{\mathrm{v}}\right)
$$

em que $\rho_{\mathrm{s}}$ é a densidade do solo $\left(\mathrm{g} \mathrm{cm}^{-3}\right)$, determinada em laboratório; $\mathrm{C}_{\mathrm{sd}}$ é o calor específico do solo seco $\left(\mathrm{J} \mathrm{kg}^{-1} \mathrm{~K}^{-1}\right)$; $\mathrm{C}_{\mathrm{sw}}$ é o calor específico da água no solo $\left(\mathrm{J} \mathrm{kg}^{-1} \mathrm{~K}^{-1}\right)$ e $\theta_{\mathrm{v}}$ é a umidade do solo $\left(\mathrm{cm}^{3} \mathrm{~cm}^{-3}\right)$, obtida pela média semanal coletada por meio de perfil de umidade do solo. O equipamento utilizado nessas medições foi a sonda PR1/6 (Delta), que mede a umidade volumétrica em diferentes profundidades no perfil do solo. A equação de calibração generalizada para solo mineral é dada a seguir:

$$
\theta_{\mathrm{v}}=0,086+0,505 \mathrm{~V}_{\text {out }}+7,81 \mathrm{~V}_{\text {out }}^{2}+32,46 \mathrm{~V}_{\text {out }}^{3}+47,96 \mathrm{~V}_{\text {out }}^{4}
$$

em que $\mathrm{V}_{\text {out }}$ é a umidade do solo expressa em Volts.

\section{Calor armazenado pelo dossel vegetativo}

O calor armazenado pelo dossel da mangueira foi calculado com base em medidas do gradiente de temperatura do ar, em três níveis espaçados 1,0 m a partir da superfície do solo, totalizando duas camadas no interior do dossel vegetativo da mangueira, pela equação (McCaughey, 1985):

$$
\mathrm{S}_{\mathrm{d}}=\frac{\left(\sum \rho_{\mathrm{a}} \mathrm{C}_{\mathrm{p}} \Delta \mathrm{h}\left(\frac{\mathrm{T}_{\mathrm{i}}+\mathrm{T}_{\mathrm{i}+1}}{2}\right)\right)_{\mathrm{j}+1}-\left(\sum \rho_{\mathrm{a}} \mathrm{C}_{\mathrm{p}} \Delta \mathrm{h}\left(\frac{\mathrm{T}_{\mathrm{i}}+\mathrm{T}_{\mathrm{i}+1}}{2}\right)\right)_{\mathrm{j}}}{1200}
$$

em que, $\rho_{\mathrm{ar}}$ é a densidade do ar $\left(1,3 \mathrm{~kg} \mathrm{~m}^{-3}\right), \Delta$ h é distância vertical entre os sensores de medidas ( $1 \mathrm{~m}), \mathrm{T}_{\mathrm{i}}$ é a temperatura do ar nas alturas “i” e "j” que representam cada instante em que se calculou a temperatura média da camada de ar; assim, se obteve a energia térmica do ar, nesse instante, em cada uma das camadas do dossel vegetativo. O calor armazenado no dossel do pomar de mangueiras foi obtido pela diferença entre as somas das energias térmica de cada uma das camadas, estimadas entre cada intervalo de 20 min, dividindo-se o resultado pelo tempo em segundos, ou seja, $1200 \mathrm{~s}$.

\section{Análise estatística}

Os valores médios das densidades de fluxos de energia $\left(R_{n}, L E, H\right.$, e $\left.G\right)$ e da evapotranspiração $\left(E_{c}\right)$ do pomar de mangueiras, obtidos com base no calor armazenado, acima das placas de fluxo de calor no solo, e sem se considerar o armazenamento desse calor acima do fluxímetro, foram avaliados pelo teste t-Student em nível de 5\% de probabilidade. Estabeleceu-se, como hipótese nula, aquela em que não existe diferença significativa entre as médias e como hipótese alternativa, aquela em que, efetivamente, existiria diferença significativa entre as médias nesse nível de probabilidade. A estatística do teste é a seguinte:

$$
\mathrm{t}=\left|\mu_{1}-\mu_{2}\right| / \sqrt{\frac{\mathrm{m}}{\left(\sigma_{1}^{2}+\sigma_{2}^{2}\right)}}
$$

em que m é o tamanho da amostra de dados da variável micrometeorológica; $\mu_{1}$ e $\sigma_{1}{ }^{2}$ são, respectivamente, a média aritmética e a variância da variável micrometeorológica obtida com base no calor armazenado acima dos fluxímetros; $\mu_{2}$ e $\sigma_{2}{ }^{2}$ são, respectivamente, a média aritmética e a variância da variável micrometeorológica obtida sem se considerar o calor armazenado acima dos fluxímetros. Ao nível de $5 \%$ de probabilidade, se a estatística t for menor que 1,96 , acata-se a hipótese nula de que não há diferença significativa entre as médias. Por outro lado, se t for maior que 1,96, rejeita-se a hipótese nula; logo, as médias são estatisticamente diferentes a nível de 5\% de probabilidade.

\section{RESULTADOS E DISCUSSÃO}

\section{Partição dos componentes do balanço de energia}

A partição do saldo de radiação $\left(R_{n}\right)$ nos demais componentes do balanço de energia, para 14 dias não-consecutivos do período experimental, com correção $\left(G_{c c}\right)$ e sem correção do fluxo de calor no solo $\left(\mathrm{G}_{\mathrm{sc}}\right)$, é apresentada na Tabela 1. Quando o balanço de energia foi realizado com 
Tabela 1. Partição do saldo de radiação $\left(R_{n}\right)$ em fluxos de calor latente $(L E)$, calor sensível para o ar $(H)$ e fluxo de calor do solo com correção( $\left.G_{c c}\right)$ e sem correção $\left(\mathrm{G}_{\mathrm{sc}}\right)$ e calor armazenado no dossel da mangueira $\left(\mathrm{S}_{\mathrm{d}}\right)$

\begin{tabular}{|c|c|c|c|c|c|c|c|c|}
\hline \multirow{2}{*}{ Data } & \multicolumn{4}{|c|}{$\mathrm{G}_{\mathrm{cc}}(\%)$} & \multicolumn{4}{|c|}{$\mathrm{G}_{\mathrm{sc}}(\%)$} \\
\hline & $L E / R_{n}$ & $G_{c} / R_{n}$ & $H / R_{n}$ & $S_{d} / R_{n}$ & $\mathrm{LE} / \mathrm{R}_{\mathrm{n}}$ & $G / R_{n}$ & $H / R_{n}$ & $S_{d} / R_{n}$ \\
\hline 01/5/2005 & 69,18 & 5,47 & 25,52 & $-0,17$ & 73,66 & 0,89 & 25,63 & $-0,17$ \\
\hline $04 / 5 / 2005$ & 69,09 & 9,41 & 21,61 & $-0,11$ & 76,23 & 1,49 & 22,38 & $-0,10$ \\
\hline 05/5/2005 & 62,33 & 4,54 & 33,23 & $-0,11$ & 62,33 & 4,54 & 33,23 & $-0,11$ \\
\hline 06/5/2005 & 65,75 & 3,36 & 31,01 & $-0,13$ & 67,03 & 1,15 & 31,94 & $-0,13$ \\
\hline $14 / 5 / 2005$ & 56,10 & 4,40 & 39,62 & $-0,13$ & 58,54 & 0,69 & 40,89 & $-0,13$ \\
\hline $15 / 5 / 2005$ & 87,26 & 8,99 & 3,92 & $-0,18$ & 95,52 & 0,27 & 3,92 & $-0,18$ \\
\hline $30 / 6 / 2005$ & 85,26 & 6,26 & 8,68 & $-0,20$ & 89,57 & 0,95 & 9,67 & $-0,20$ \\
\hline 01/7/2005 & 85,35 & 6,70 & 8,12 & $-0,17$ & 85,35 & 6,70 & 8,12 & $-0,17$ \\
\hline 04/7/2005 & 85,46 & 8,62 & 6,09 & $-0,17$ & 92,76 & 0,84 & 6,56 & $-0,17$ \\
\hline 05/7/2005 & 84,32 & 7,34 & 8,49 & $-0,14$ & 90,85 & 0,19 & 9,10 & $-0,14$ \\
\hline 18/7/2005 & 86,36 & 6,41 & 7,46 & $-0,23$ & 91,14 & 0,51 & 8,58 & $-0,23$ \\
\hline 06/8/2005 & 87,64 & 10,59 & 1,96 & $-0,18$ & 97,59 & 0,42 & 2,18 & $-0,18$ \\
\hline 08/8/2005 & 87,78 & 11,05 & 1,37 & $-0,20$ & 97,91 & 0,73 & 1,56 & $-0,20$ \\
\hline 09/8/2005 & 87,24 & 11,25 & 1,71 & $-0,20$ & 97,82 & 0,47 & 1,91 & $-0,20$ \\
\hline Média & 78,51 & 7,46 & 14,20 & $-0,16$ & 84,02 & 1,42 & 14,69 & $-0,16$ \\
\hline Desvio-padrão & 11,30 & 2,58 & 13,19 & 0,04 & 13,32 & 1,79 & 12,87 & 0,04 \\
\hline
\end{tabular}

$\mathrm{G}_{\mathrm{cc}}$, a partição média de $\mathrm{R}_{\mathrm{n}}$ nas demais densidades de fluxos foi representada da seguinte forma: $L E / R_{n}=78,51 \%$; $\mathrm{G}_{\mathrm{cc}} / \mathrm{R}_{\mathrm{n}}=7,46 \% ; \mathrm{H} / \mathrm{R}_{\mathrm{n}}=14,20 \%$ e $\mathrm{S}_{\mathrm{d}} / \mathrm{R}_{\mathrm{n}}=0,16 \%$, enquanto com $G_{s c}$ a partição representou: $L E / R_{n}=84,02 \%$; $\mathrm{G}_{\mathrm{sc}} / \mathrm{R}_{\mathrm{n}}=1,42 \% ; \mathrm{H} / \mathrm{R}_{\mathrm{n}}=14,69 \%$ e $\mathrm{S}_{\mathrm{d}} / \mathrm{R}_{\mathrm{n}}=0,16 \%$, respectivamente.

Pode-se observar, portanto, que o calor armazenado no dossel da mangueira apresentou pequena contribuição $(0,16 \%)$ ao cálculo do balanço de energia; constatou-se também que $H / R_{n}$ foi basicamente igual nos dois casos: $14,20 \%$ com $G_{\text {cc }}$ e $14,69 \%$ com $G_{\text {sc }}$; entretanto, a proporção de $R_{n}$ convertido em fluxo de calor sensível no solo corrigido $\left(\mathrm{G}_{\mathrm{cc}}=7,46 \%\right)$ foi bastante superior àquela nãocorrigida $\left(\mathrm{G}_{\mathrm{sc}}=1,42 \%\right)$. A média da proporção $\mathrm{S}_{\mathrm{d}} / \mathrm{R}_{\mathrm{n}}$, ao longo do ciclo produtivo do pomar de mangueiras permaneceu constante, enquanto a proporção $H / R_{n}$ foi muito próxima nas duas condições de elaboração do balanço de energia. Independentemente da forma de cálculo do balanço de energia (com ou sem correção), o menor consumo do saldo de radiação, na forma de calor latente, ocorreu nas fases inicial e intermediária do ciclo produtivo da mangueira, enquanto o maior consumo se deu na fase da maturação; resultados semelhantes foram encontrados por Mokate et al. (1995) para a cultura de trigo e por Azeve- do et al. (2003) em pomar de mangueiras. O consumo de $R_{n}$ na forma de LE, considerando-se a correção $\left(G_{c c}\right)$ foi, em média, 6,55\% inferior àquele obtido sem a correção do fluxo de calor no solo $\left(\mathrm{G}_{\mathrm{sc}}\right)$, cujos resultados sugerem que o fluxo de calor latente é afetado pelo fluxo de calor no solo, porém o fluxo de calor sensível varia pouco e o calor armazenado no dossel vegetativo permanece inalterado, considerando-se ou não o calor armazenado acima dos fluxímetros.

Os valores médios dos componentes do balanço de energia para dias representativos de cada fase fenológica do pomar de mangueiras, com correção $\left(G_{c c}\right)$ e sem correção do fluxo de calor no solo $\left(G_{\mathrm{sc}}\right)$ são apresentados na Tabela 2.

Os valores médios do saldo de radiação e do fluxo de calor latente apresentaram comportamento crescente ao longo do ciclo produtivo do pomar de mangueiras, ao passo que o fluxo de calor sensível se mostrou decrescente, exceto na fase fenológica de queda de frutos. Evidentemente, nas duas formas de cálculo do balanço de energia as médias de $R_{n}$ e $S_{d}$ permaneceram inalteradas, mas as médias das demais densidades de fluxo foram diferentes, especialmente aquelas referentes ao fluxo de calor no solo com correção $\left(23,7 \mathrm{~W} \mathrm{~m}^{-2}\right)$ e sem correção $\left(2,0 \mathrm{~W} \mathrm{~m}^{-2}\right)$.

Tabela 2. Valores médios dos componentes do balanço de energia $\left(\mathrm{W} \mathrm{m}^{-2}\right)$ : saldo de radiação $\left(\mathrm{R}_{\mathrm{n}}\right)$, fluxo de calor latente (LE), fluxo de calor sensível $(H)$, calor armazenado no dossel da planta $\left(S_{d}\right)$ e fluxo de calor no solo, com correção $\left(G_{c c}\right)$ e sem correção $\left(G_{s c}\right)$, para dias representativos de cada fase fenológica do pomar de mangueiras

\begin{tabular}{|c|c|c|c|c|c|c|c|c|c|c|}
\hline \multirow{2}{*}{ Fase fenológica } & \multicolumn{5}{|c|}{ Com correção (G } & \multicolumn{5}{|c|}{ Sem correção $\left(G_{s c}\right)$} \\
\hline & $R_{n}$ & LE & H & $\mathrm{G}_{\mathrm{cc}}$ & $S_{d}$ & $\mathbf{R}_{\mathbf{n}}$ & LE & H & $\mathrm{G}_{\mathrm{sc}}$ & $S_{d}$ \\
\hline Floração de frutos & 209,7 & $-139,2$ & $-58,6$ & $-12,1$ & 0,3 & 209,7 & $-147,0$ & $-60,3$ & $-2,6$ & 0,3 \\
\hline Queda de frutos & 267,8 & $-183,2$ & $-68,4$ & $-16,6$ & 0,4 & 267,8 & $-196,9$ & $-70,5$ & $-1,4$ & 0,4 \\
\hline Formação de frutos & 329,3 & $-282,6$ & $-22,2$ & $-25,2$ & 0,6 & 329,3 & $-303,8$ & $-24,4$ & $-1,8$ & 0,6 \\
\hline Maturação & 364,7 & $-319,1$ & $-5,6$ & $-40,7$ & 0,7 & 364,7 & $-365,9$ & $-6,4$ & $-2,0$ & 0,7 \\
\hline Média & 292,9 & $-231,0$ & $-38,7$ & $-23,7$ & 0,5 & 292,9 & $-253,4$ & $-40,4$ & $-2,0$ & 0,5 \\
\hline Desvio-padrão & 68,4 & 83,9 & 29,7 & 12,6 & 0,2 & 68,4 & 99,5 & 30,1 & 0,5 & 0,2 \\
\hline
\end{tabular}




\section{Evapotranspiração do pomar de mangueiras}

A Tabela 3 apresenta os valores médios diários da evapotranspiração, fluxo de calor no solo com correção $\left(G_{\mathrm{cc}}\right)$, fluxo de calor no solo sem correção $\left(\mathrm{G}_{\mathrm{sc}}\right)$ e calor armazenado no dossel da planta $\left(\mathrm{S}_{\mathrm{d}}\right)$, para 14 dias do período experimental. As médias e os desvios-padrão do fluxo de calor no solo com e sem correção foram, nesse período: $22,44 \pm 11,14 \mathrm{~W} \mathrm{~m}^{-2}$ e $2,01 \pm 0,86 \mathrm{~W} \mathrm{~m}^{-2}$, respectivamente; desta forma, $\mathrm{G}_{\mathrm{sc}}$ representou uma pequena fração do $G_{c c}$, ou seja, apenas 7,7\%, A média do fluxo de calor armazenado no dossel vegetativo das plantas foi $0,49 \pm 0,20 \mathrm{~W} \mathrm{~m}^{-2}$. As médias e os desvios-padrão da evapotranspiração do pomar de mangueiras com base no fluxo de calor no solo com correção, $\mathrm{ET}_{\mathrm{c}}\left(\mathrm{G}_{\mathrm{cc}}\right)$ e sem correção, $\mathrm{ET}_{\mathrm{c}}\left(\mathrm{G}_{\mathrm{sc}}\right)$ para os 14 dias do período experimental, foram 3,2 $\pm 1,03$ e 3,4 $\pm 1,22 \mathrm{~mm} \mathrm{~d}^{-1}$, respectivamente. Em todos os dias analisados a $E T_{\mathrm{C}}\left(\mathrm{G}_{\mathrm{cc}}\right)$ foi inferior a $\mathrm{ET}_{\mathrm{C}}\left(\mathrm{G}_{\mathrm{sc}}\right)$, bem como na média do período, exceto no dia 14/05/2005.

Tabela 3. Evapotranspiração diária $\left(E T_{c}\right)$ do pomar de mangueiras considerando-se o fluxo de calor no solo com correção - $E T_{c}\left(G_{c c}\right)$ e sem correção - $\mathrm{ET}_{\mathrm{c}}\left(\mathrm{G}_{\mathrm{sc}}\right)$ e os valores médios diários do fluxo de calor no solo com correção $\left(\mathrm{G}_{c c}\right)$ e sem correção $\left(\mathrm{G}_{\mathrm{sc}}\right)$ e o calor armazenado no dossel da planta $\left(S_{d}\right)$ para 14 dias, durante o período experimental

\begin{tabular}{|c|c|c|c|c|c|}
\hline DIA & $\begin{array}{c}\mathrm{ET}_{\mathrm{c}}\left(\mathrm{G}_{\mathrm{cc}}\right) \\
\mathrm{mm} \mathrm{d}^{-1}\end{array}$ & $\begin{array}{l}\mathrm{ETc}\left(\mathrm{G}_{\mathrm{sc}}\right) \\
\mathrm{mm} \mathrm{d}^{-1}\end{array}$ & $\begin{array}{c}G_{c c} \\
W m^{-2}\end{array}$ & $\begin{array}{c}G_{\text {sc }} \\
W^{-2}\end{array}$ & $\begin{array}{c}S_{d} \\
W m^{-2}\end{array}$ \\
\hline 01/5/2005 & 1,6 & 1,7 & $-8,74$ & $-1,42$ & 0,28 \\
\hline 04/5/2005 & 2,2 & 2,5 & $-21,76$ & $-3,45$ & 0,25 \\
\hline 05/5/2005 & 2,1 & 2,1 & $-10,50$ & $-3,02$ & 0,25 \\
\hline 06/5/2005 & 2,0 & 2,0 & $-7,28$ & $-2,50$ & 0,27 \\
\hline $14 / 5 / 2005$ & 2,6 & 1,9 & $-14,28$ & $-2,25$ & 0,42 \\
\hline $15 / 6 / 2005$ & 2,4 & 2,6 & $-19,00$ & $-0,56$ & 0,37 \\
\hline 30/6/2005 & 4,0 & 4,2 & $-21,12$ & $-3,21$ & 0,66 \\
\hline 01/7/2005 & 3,9 & 4,2 & $-23,00$ & $-1,72$ & 0,58 \\
\hline 04/7/2005 & 2,8 & 3,0 & $-20,45$ & $-2,00$ & 0,40 \\
\hline $05 / 7 / 2005$ & 3,8 & 4,1 & $-23,83$ & $-0,62$ & 0,47 \\
\hline $18 / 7 / 2005$ & 4,1 & 4,3 & $-22,81$ & $-1,80$ & 0,81 \\
\hline 06/8/2005 & 4,3 & 4,8 & $-39,95$ & $-1,58$ & 0,70 \\
\hline 08/8/2005 & 4,3 & 4,8 & $-39,49$ & $-2,29$ & 0,70 \\
\hline 09/8/2005 & 4,5 & 5,0 & $-41,90$ & $-1,75$ & 0,74 \\
\hline Média & 3,2 & 3,4 & $-22,44$ & 2,01 & 0,49 \\
\hline Desvio-padrão & 1,03 & 1,22 & 11,14 & 0,86 & 0,20 \\
\hline
\end{tabular}

A Tabela 4 exibe a evapotranspiração e os valores médios do fluxo de calor no solo e calor armazenado no dossel da planta, para cada fase fenológica do pomar de mangueiras. Os valores médios e os desvios-padrão de $\mathrm{G}_{\mathrm{sc}}, \mathrm{G}_{\mathrm{cc}}$ e $S_{d}$ foram -18,71 $\pm 5,63 ;-1,86 \pm 0,52$ e $0,44 \pm 0,15 \mathrm{~W} \mathrm{~m}^{-2}$, respectivamente; portanto, a ordem de grandeza desses valores é semelhante àquela obtida para a média de 14 dias do período experimental. As médias e os desvios-padrão de $\operatorname{ET}_{\mathrm{c}}\left(\mathrm{G}_{\mathrm{cc}}\right)$ e $\mathrm{ET}_{\mathrm{c}}\left(\mathrm{G}_{\mathrm{sc}}\right)$ foram muito próximos daqueles obtidos para os 14 dias do período experimental, indicando a boa representatividade dos dias selecionados para as fases fenológicas. Em ambas as análises, os valores médios de $\operatorname{ET}_{\mathrm{c}}\left(\mathrm{G}_{\mathrm{cc}}\right)$ e $\mathrm{ET}_{\mathrm{c}}\left(\mathrm{G}_{\mathrm{sc}}\right)$ não foram estatisticamente diferentes, de acordo com o teste t-Student a nível de 5\% de probabilidade. Os valores médios da evapotranspiração $\left(\mathrm{ET}_{\mathrm{C}}\right)$ foram bastante próximos dos obtidos por Azevedo et al.
Tabela 4. Evapotranspiração e valores médios do fluxo de calor no solo com correção $\left(\mathrm{G}_{\mathrm{cc}}\right)$ e sem correção $\left(\mathrm{G}_{\mathrm{sc}}\right)$ do fluxo de calor no solo e calor armazenado no dossel vegetativo $\left(S_{d}\right)$ para cada fase fenológica do pomar de mangueiras

\begin{tabular}{|c|c|c|c|c|c|c|}
\hline \multirow{2}{*}{ Fase fenológica } & \multirow{2}{*}{ Data } & $G_{c c}$ & $G_{s c}$ & $S_{d}$ & $\mathrm{ET}_{\mathrm{c}}\left(\mathrm{G}_{\mathrm{cc}}\right)$ & $\mathrm{ET}_{\mathrm{c}}\left(\mathrm{G}_{\mathrm{sc}}\right)$ \\
\hline & & \multicolumn{3}{|c|}{$W m^{-2}$} & \multicolumn{2}{|c|}{$\mathrm{mm} \mathrm{d}^{-1}$} \\
\hline Floração de frutos & 17/04-07/05 & $-12,07$ & $-2,60$ & 0,26 & 1,98 & 2,08 \\
\hline Queda de frutos & 08/05-17/06 & $-16,64$ & $-1,41$ & 0,40 & 2,50 & 2,25 \\
\hline Formação de frutos & $18 / 06-07 / 08$ & $-25,19$ & $-1,82$ & 0,60 & 3,72 & 3,96 \\
\hline Maturação de frutos & 08/08-18/08 & $-40,70$ & $-2,00$ & 0,70 & 4,40 & 4,90 \\
\hline Média & - & $-18,71$ & $-1,86$ & 0,44 & 3,15 & 3,30 \\
\hline Desvio-padrão & - & 5,63 & 0,52 & 0,15 & 1,12 & 1,37 \\
\hline
\end{tabular}

(2003), para experimento de campo em pomar de mangueiras; nesta pesquisa se obteve uma evapotranspiração média diária que variou de $3,0 \mathrm{~mm} \mathrm{~d}^{-1}$, na fase de floração para $5,5 \mathrm{~mm} \mathrm{~d}^{-1}$ na fase de formação de frutos.

Os valores médios dos componentes do balanço de energia para os dois períodos analisados (14 dias do período experimental e dias representativos dos estágios fenológicos), exceto para os fluxos de calor no solo, não apresentaram diferença estatisticamente significativa a nível de 5\% de probabilidade pelo teste t-Student. De acordo com o teste t-Student, os valores médios de $G_{\mathrm{cc}}$ e $G_{\mathrm{sc}}$ para os 14 dias do período experimental e as médias desses valores dos estágios fenológicos são estatisticamente diferentes a nível de $5 \%$ de probabilidade.

A Figura 1 exibe o comportamento do fluxo de calor no solo sem correção $\left(\mathrm{G}_{\mathrm{sc}}\right)$ e com correção $\left(\mathrm{G}_{\mathrm{cc}}\right)$ e a variação do fluxo
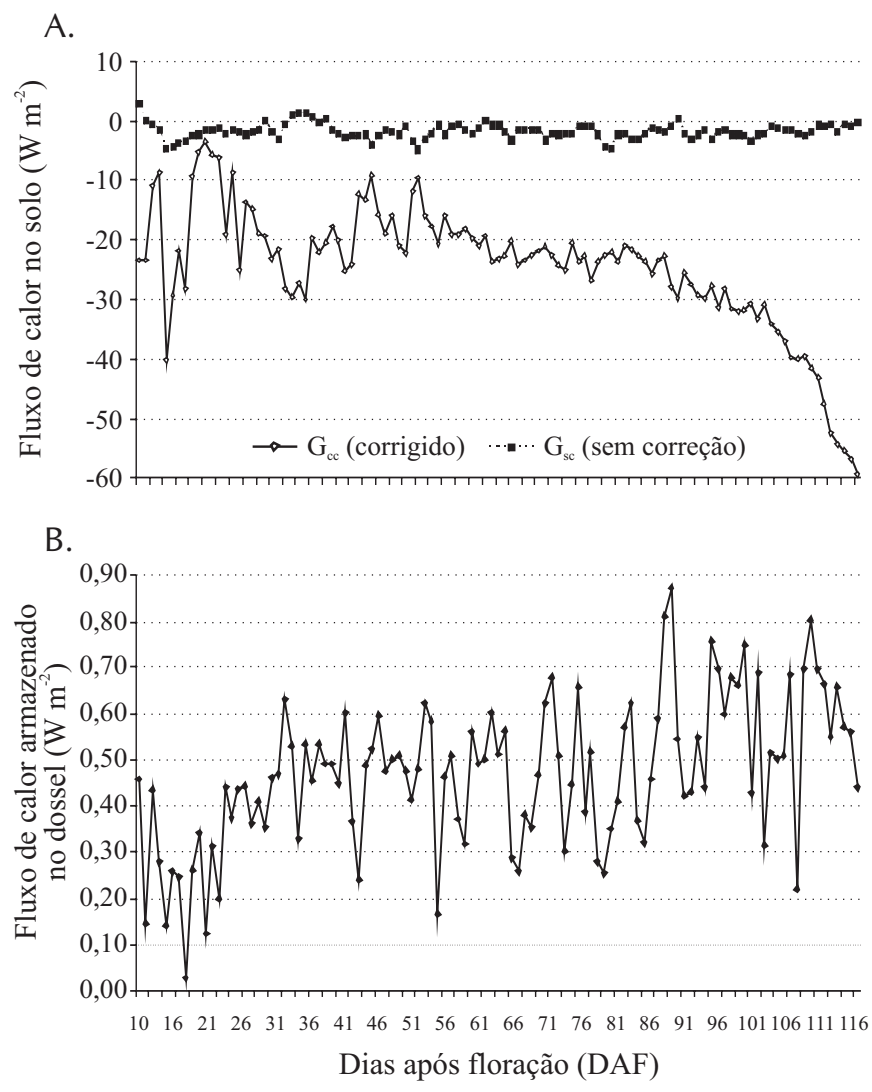

Figura 1. Comportamento do fluxo de calor no solo sem correção $\left(\mathrm{G}_{\mathrm{sc}}\right)$ e com correção $\left(\mathrm{G}_{\mathrm{cc}}\right)(\mathrm{A})$ e o fluxo de calor armazenado no dossel das plantas $\left(\mathrm{S}_{\mathrm{d}}\right)$, ao longo do ciclo produtivo do pomar de mangueiras $(\mathrm{B})$ 
de calor armazenado no dossel das plantas ao longo do ciclo produtivo do pomar de mangueiras. Observa-se que $G_{c c}$ se manteve estacionário durante todo o período analisado enquanto $\mathrm{G}_{\mathrm{sc}}$ exibiu grande variabilidade, com comportamento decrescente no final do ciclo produtivo da mangueira (Figura 1A). Quanto ao fluxo de calor armazenado no dossel das plantas, constatamse valores muito pequenos, inferiores a $1 \mathrm{~W} \mathrm{~m}^{-2}$, durante todo o período analisado (Figura $1 \mathrm{~B}$ ).

Este resultado indica que o fluxo de calor armazenado no dossel da planta exerceu pouca influência no cálculo do balanço de energia do pomar de mangueiras nas condições de realização deste experimento. Os valores apresentados por este fluxo indicam tendência crescente ao longo do ciclo produtivo do pomar de mangueiras, possivelmente em razão do aumento da demanda atmosférica na região, no mesmo período.

\section{CONCLUSÕES}

1. O efeito do calor armazenado no dossel da mangueira irrigada é irrelevante quando comparado com outros componentes do balanço de energia em pomar de mangueiras.

2. O calor armazenado acima das placas de calor no solo não interfere significativamente no cálculo dos fluxos de calor sensível e latente nem na evapotranspiração da mangueira irrigada na região do submédio Rio São Francisco.

3. O fluxo de calor no solo, corrigido com o calor armazenado acima dos fluxímetros, difere estatisticamente, em nível de $5 \%$ de probabilidade, daquele obtido sem a correção.

\section{LITERATURA CITADA}

Angus, D. E.; Watts, P. J. Evapotranspiration: how good is the Bowen ratio method. Agricultural Water Management, v.8, n.1, p.133-150, 1984.

Azevedo, P. V.; Silva, B. B.; Silva, V. P. R. Water requirements of irrigated mango orchard in northeast Brazil. Agricultural Water management, v.58, n.1, p.241-254, 2003.

Bowen, I. S. The ratio of heat losses by conduction and by evaporation from any water surface. Physical Review, v.27, p.779-787, 1926.

Cunha, G. R.; Bergamaschi, H.; Berlato, M. A. Balanço de energia em cultura de milho, Revista Brasileira de Agrometeorologia, v.4, n.1, p.1-14, 1996.
Daamen, C. C.; Dugas, W. A. Prendergast, P. T. Energy flux measurements in a sheltered lemon orchard. Agricultural and Forest Meteorology, v.93, n.1, p.171-183, 1999.

Heilman, J. L.; Mclnnes, K. J.; Savage, M. J. Soil and canopy energy in a west Texas vineyard. Agricultural and Forest Meteorology, v.71, n.1, p.99-114, 1994.

Kustas, W. P.; Prueger, J. H.; Hatfield, J. L.; Ramalingam, H.; Hipps, L. E. Variability in soil heat flux from a mesquite dune site. Agricultural and Forest Meteorology, v.103, n.1, p.249-264, 2000.

McCaughey, J. H. Energy balance storage terms in a nature mixed forest at Petawawa, Ontario - a case study. Boundary Layer Meteorological, v.31, n.1, p.89-101, 1985.

Mokate, A. S.; Varshneya, M. C.; Naidu, T. R. V. Evapotranspiration and energy balance studies over wheat crop by Bowen ratio energy balance method. Journal Maharashtra Agricultural Universities, v.20, n.2, p.273-276, 1995.

Rosenberg, N. J.; Blad, B. L; Verma, S. B. Microclimate. The biological environment. 2. Edition, Lincoln, Nebraska: John Wiley \& Sons, 1983, 495p.

Silans, A. P.; Monteny, B. A.; Lhomme, J. P. The correction of soil heat flux measurements to derive an accurate surface energy balance by the Bowen ratio method. Journal of Hydrology, v.4, n.1, p.453-465, 1997.

Silva, V. P. R.; Azevedo, P. V.; Silva, B. B. Seasonal patterns of the energy balance components in a mango (Mangifera indica L.) orchard grown in northeast Brazil. Agricultural Journal, v.1, n.1, p.18-23, 2006.

Stannard, D. I.; Blanford, J. H.; Kustas, W. P. Interpretation of surface flux menasurements in heterogeneous terrain during the monsoon '90 experiment. Water Resources Research, v.30, n.5, p.1227-1239, 1994.

Teixeira, A. H. C. Avaliação dos componentes do balanço de energia durante o primeiro ano de cultura da banana. Revista Brasileira de Engenharia Agrícola e Ambiental, v.3, n.1, p.28-32, 2001.

Trambouze, W.; Bertuzzi, P.; Voltz, M. Comparison of methods for estimating actual evapotranspiration in a row-cropped vineyard. Agricultural and Forest Meteorology, v.91, p.193-208, 1998.

Unland, H. E.; Houser, P.R,; Shuttleworth, W. J.; Yang, Z. L. Surface flux measurements and modelling at a semi-arid sonoran desert site. Agricultural and Forest Meteorology, v.82, n.1, p.119-153, 1996.

Wright, J. L. New evapotranspiration crop coefficient. Journal of the Irrigation and Drainage Division, v.108, n.2, p.5774, 1982. 